\title{
Oxcarbazepine in Monotherapy
}

\author{
MOGENS DAM
}

University Clinic of Neurology, Copenhagen, Denmark

\begin{abstract}
Summary
Altogether 235 patients with newly diagnosed epilepsy were randomly allocated to treatment with either oxcarbazepine (OXC) or carbamazepine (CBZ) in a double-blind multicentre trial. After a titration phase of 4-8 weeks the optimum individual dose of trial medication was determined and treatment with this dose was continued for another 48 weeks.

The results of the study indicate that there is no significant difference in seizure frequency between OXC and CBZ; no correlation was found between the therapeutic effect and EEG in either treatment group. OXC caused significantly $(p=0.04)$ fewer "severe" side-effects than CBZ. Global evaluation of tolerability showed a trend towards better tolerability of OXC. There was no correlation between either efficacy or tolerability and serum trough levels of the investigational drugs. Clinically relevant abnormal laboratory test findings were observed in two patients, both on CBZ.

In conclusion, $\mathrm{OXC}$ is a major anti-epileptic drug, which is as effective as CBZ in the treatment of partial seizures and generalized convulsions. With fewer side-effects than CBZ, it represents a valuable alternative particularly in patients who develop side-effects which prevent optimum seizure control.
\end{abstract}

\section{Introduction}

Oxcarbazepine is the keto-analogue of carbamazepine. Despite their structural similarity, these two compounds have completely different metabolic profiles.

In contrast to many animal models, in humans oxcarbazepine is rapidly and almost completely metabolized to 10,11-dihydro-10-hydroxycarbamazepine (Feldman et al., 1981). This monohydroxy metabolite is the main metabolite responsible for the anti-epileptic activity of oxcarbazepine in man.

In order to compare the efficacy and tolerability of oxcarbazepine and carbamazepine we have conducted a clinical trial with the two substances administered as monotherapy in patients with newly diagnosed epilepsy (Dam et al., 1989). 


\section{Design}

A total of 235 patients from 20 centres in Denmark, Finland, Norway, and Sweden was included in the study. The trial was designed as a double-blind, between-patient, multicentre study with patients randomly allocated to treatment with either oxcarbazepine ( ${ }^{\circledR}$ Trileptal) or carbamazepine ( ${ }^{\circledR}$ Tegretol). After a titration phase of 4 to 8 weeks, the treatment was continued using the optimum dose for 48 weeks. To ensure a proper baseline, all seizures and side-effects during the last 4 weeks preceding the start of the trial were recorded.

\section{Patients}

The patients were men or women aged 15-65 years, suffering from newly diagnosed and previously untreated epilepsy. Only patients with primarily generalized seizures (tonic-clonic seizures) and those with partial seizures with or without secondary generalization were included. We excluded women who were pregnant or were trying to become pregnant, patients with known heart, liver, kidney, and thyroid diseases, patients with abnormally low leukocyte or platelet counts, inoperable tumours, or known hypersensitivity to tricyclic antidepressants, and patients who were being treated with drugs known to interact with carbamazepine.

\section{Criteria of Assessment}

The following criteria were used to assess therapeutic efficacy: changes in seizure frequency;

changes in electroencephalogram (EEG) recordings with regard to epileptic activity between baseline and the end of the maintenance period;

global evaluation of therapeutic efficacy at the end of the maintenance period.

The criteria used to assess tolerability were subjective symptoms reported by the patient at each visit, neurological signs observed at each visit, and laboratory tests. Blood pressure, pulse rate, and trough plasma concentration of carbamazepine and 10,11-dihydro-10-hydroxycarbamazepine were monitored at every visit.

Of the 235 patients, 41 were excluded, seven because of protocol violations, 10 owing to poor compliance, and 24 for administrative reasons. Forty-two patients discontinued the trial prematurely due to either insufficient efficacy (carbamazepine: 2; oxcarbazepine: 2) or poor tolerability (carbamazepine: 25; oxcarbazepine: 13). All patients who withdrew from the study because of insufficient efficacy were included in the analysis of efficacy, and all who dropped out because of poor tolerability were included in the analysis of tolerability. A total of 190 patients (carbamazepine: 98 ; oxcarbazepine: 92) was included in the analysis of tolerability. Out of the 38 patients who discontinued the trial because of poor tolerability, 29 withdrew during the titration phase and were therefore not included in the analysis of efficacy. 


\section{Results}

Table 1 shows the number of seizures during the baseline period, compared with the number of seizures during treatment with either carbamazepine or oxcarbazepine. During the maintenance phase $81.4 \%$ of patients treated with carbamazepine had at leasta $50 \%$ reduction in seizure frequency, versus $80.2 \%$ of patients on oxcarbazepine. Analyses of efficacy in different types of seizure did not show any statistically significant differences between the two drugs.

TABLE 1. Number of seizures/month before and during treatment with oxcarbazepine $(O X C)$ and carbamazepine (CBZ)

\begin{tabular}{lllllllllll}
\hline Drug & $\begin{array}{l}\text { No. of } \\
\text { of patients }\end{array}$ & Baseline & \multicolumn{5}{c}{ Number of seizures } \\
Maintenance phase
\end{tabular}

Global evaluation of efficacy was performed by the physician at the end of the maintenance phase (Table 2). No statistically significant difference between the two treatments was found $(p=0.77)$.

TABLE 2. Global evaluation of efficacy

\begin{tabular}{llllll}
\hline Drug & $\begin{array}{l}\text { No. of } \\
\text { patients }\end{array}$ & \multicolumn{4}{c}{ Efficacy (\%) } \\
\hline & & None & Minimal & Good & Excellent \\
OXC & 80 & 3 & 1 & 41 & 55 \\
CBZ & 71 & 3 & 0 & 38 & 59 \\
\hline
\end{tabular}

No data for 14 patients ( 3 OXC and $11 \mathrm{CBZ \text {) }}$

The EEG was monitored at the beginning of the trial and at the end. No correlation between the therapeutic effect and EEG changes could be detected, nor were there any clinically relevant differences between the two drugs.

Side-effects recorded at every visit were classified as either "mild" (only recorded), "moderate" (reduction of the dose), or "severe" (immediate discontinuation of the treatment). The difference in severe side-effects (carbamazepine: 25; oxcarbazepine: 13) was statistically significant in favour of oxcarbazepine $(p=0.04)$. In 16 patients on carbamazepine the drug had to be withdrawn because of allergic reactions, as compared with 9 on oxcarbazepine. The nature of other side-effects (tiredness, headache, dizziness, and ataxia) was the same in both treatment groups.

Aglobal evaluation of tolerability was assessed by the investigators at the end of the maintenance phase. Treatment was assessed as either "very good" or "good" in $73 \%$ on carbamazepine compared with $84 \%$ on oxcarbazepine. The 
treatment with carbamazepine was assessed as either "poor" or "very poor" in $27 \%$, versus $16 \%$ on oxcarbazepine $(p=0.11)$. Two patients on carbamazepine had to be withdrawn from the trial, one with leukopenia and one with a sudden rise in liver-function parameters.

No correlation was observed between the severity of side-effects and serum concentrations (Table 3).

TABLE 3. Correlation between severity of side-effects and serum concentrations of oxcarbazepine (OXC) and carbamazepine $(C B Z)(\mu \mathrm{mol} / \mathrm{l})$

\begin{tabular}{lcccc}
\hline Drug & \multicolumn{2}{c}{ Severity } & Moderate & Severe \\
\hline OXC & None & Mild & & \\
Median & 58.0 & 57.0 & 61.0 & 55.0 \\
Mean & 61.7 & 64.8 & 67.2 & 62.0 \\
SD & 21.8 & 25.7 & 25.9 & 33.7 \\
CBZ & & & & \\
Median & 30.0 & 30.0 & 32.0 & 29.0 \\
Mean & 31.0 & 30.2 & 33.8 & 29.3 \\
SD & 10.5 & 8.5 & 11.2 & 7.3 \\
\hline
\end{tabular}

\section{Conclusion}

We thus concluded that oxcarbazepine caused significantly fewer severe side-effects requiring withdrawal from the trial than carbamazepine. The two compounds proved to be comparable with regard to efficacy. A reduction in seizure frequency of at least $50 \%$ was achieved in approximately $80 \%$ of the patients, whether on oxcarbazepine or carbamazepine.

With these facts in mind it is reasonable to conclude that oxcarbazepine should be used as the first drug of choice in the treatment of partial seizures, whether simple or complex, with or without secondary generalization. Only if oxcarbazepine turns out to be ineffective or to cause allergic reactions or other side-effects, should carbamazepine be tried. Oxcarbazepine is thus a major anti-epileptic drug which should be regarded as a viable alternative for first-line therapy.

\section{References}

Feldmann, K.E., Dörhöfer, G., Faigle, J.W. and Imhof, P. (1981). Pharmacokinetics and metabolism of GP 47 779, the main human metabolite of oxcarbazepine (GP 47680 ) in animals and healthy volunteers. In "Advances in Epileptology: XIIth Epilepsy International Symposium." (Eds M. Dam, L. Gram and J.R. Penry), pp. 89-96. Raven Press, New York.

Dam, M., Ekberg, R., Loyning, Y., Waltimo, O. and Jakobsen, K. (The Scandinavian Oxcarbazepine Study Group) (1989). A double-blind study comparing oxcarbazepine and carbamazepine in patients with newly diagnosed previously untreated epilepsy. Epilepsy Research, 3, 70-76. 


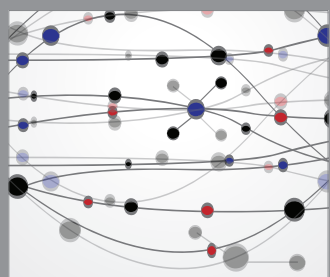

The Scientific World Journal


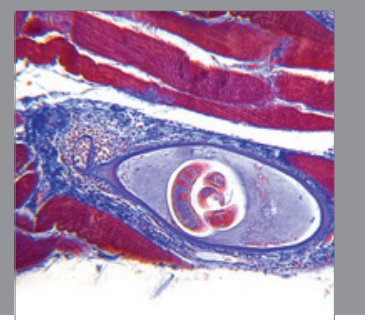

Gastroenterology

Research and Practice
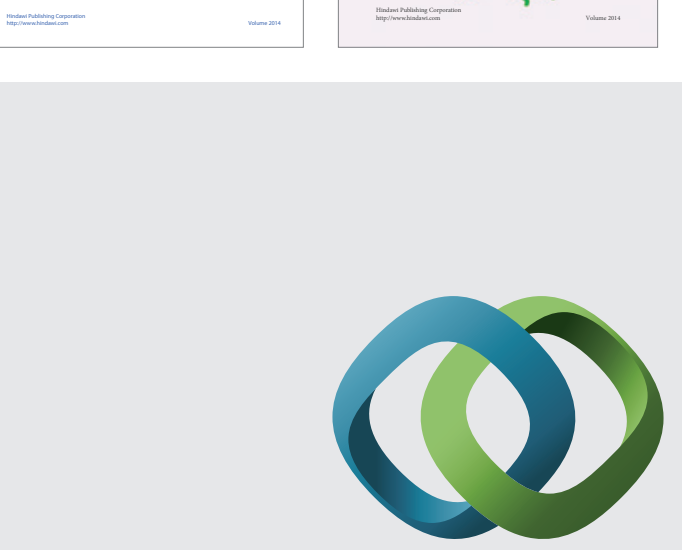

\section{Hindawi}

Submit your manuscripts at

http://www.hindawi.com
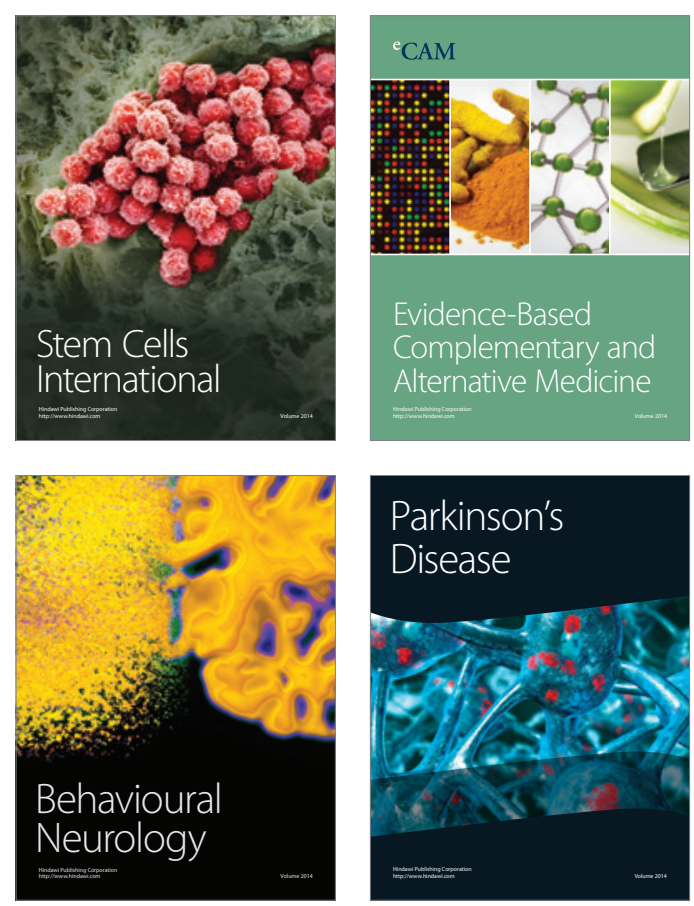



Journal of
Diabetes Research

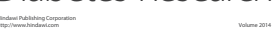

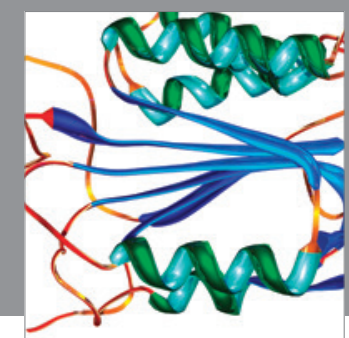

Disease Markers
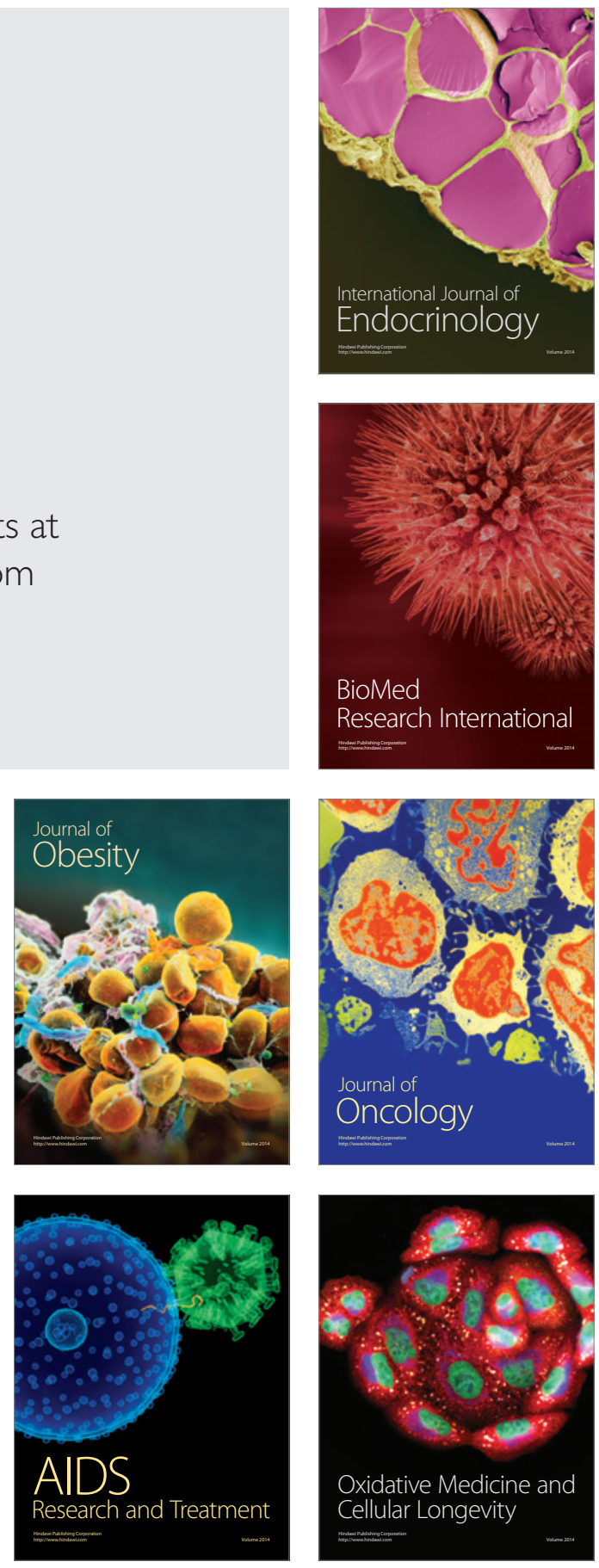